\title{
TORIC IDEALS AND DIAGONAL 2-MINORS
}

\author{
ANARGYROS KATSABEKIS
}

\begin{abstract}
Let $G$ be a simple graph on the vertex set $\{1, \ldots, n\}$ with $m$ edges. An algebraic object attached to $G$ is the ideal $P_{G}$ generated by diagonal 2minors of an $n \times n$ matrix of variables. In this paper we prove that if $G$ is bipartite, then every initial ideal of $P_{G}$ is generated by squarefree monomials of degree at most $\left\lfloor\frac{m+n+1}{2}\right\rfloor$. Furthermore, we completely characterize all connected graphs $G$ for which $P_{G}$ is the toric ideal associated to a finite simple graph. Finally we compute in certain cases the universal Gröbner basis of $P_{G}$.
\end{abstract}

\section{INTRODUCTION}

Let $X=\left(x_{i j}\right)$ be an $n \times n$ matrix of variables and $R=K\left[x_{i j} \mid 1 \leq i, j \leq n\right]$ be the polynomial ring in $n^{2}$ variables over a field $K$. For $1 \leq i<j \leq n$ we denote by $f_{i j}$ the diagonal 2-minor of $X$ given by the elements that stand at the intersection of the rows $i, j$ and the columns $i, j$, in other words, $f_{i j}=x_{i i} x_{j j}-x_{i j} x_{j i}$. Thus $f_{i j}$ is a binomial in $R$, namely a difference of two monomials.

Let $G$ be a simple graph on the vertex set $\{1, \ldots, n\}$ with the edge set $E(G)$. By a simple graph $G$ we mean an undirected graph without loops or multiple edges. Let $m$ be the cardinality of $E(G)$. We write $S$ for the polynomial ring

$$
S:=K\left[\left\{x_{i j}, x_{j i} \mid\{i, j\} \in E(G)\right\} \cup\left\{x_{i i}\right\}_{1 \leq i \leq n}\right]
$$

in $2 m+n$ variables over $K$. Let $P_{G}$ be the ideal of $S$ generated by all the binomials $f_{i j}$, where $\{i, j\}$ is an edge of $G$.

Ideals generated by diagonal 2 -minors were studied for the first time in [5]. More specifically, the authors considered the ideal $P_{G}^{\prime}=P_{G} \cdot R$ and proved that it is a complete intersection prime ideal of height $m$. They also noticed that the set of all binomials $f_{i j}$, where $\{i, j\}$ is an edge of $G$, is the reduced Gröbner basis of $P_{G}^{\prime}$ with respect to the reverse lexicographical order given by the natural ordering of variables

$x_{11}>x_{12}>\cdots>x_{1 n}>x_{21}>x_{22}>\cdots>x_{2 n}>\cdots>x_{n 1}>\cdots>x_{n n}$.

By Theorem 1.2 in [5], the initial ideal of $P_{G}^{\prime}$ with respect to the lexicographic term order given by the natural order of indeterminates is generated by squarefree monomials of degree at most 4 .

The universal Gröbner basis of an ideal $I$ generated by binomials, denoted by $U(I)$, is the union of all its reduced Gröbner bases. Universal Gröbner bases were used in integer programming and algebraic statistics to compute Markov bases of

2000 Mathematics Subject Classification. 13F20, 13P10, 05 C25.

Key words and phrases. Toric ideals, diagonal 2-minors, Gröbner bases.

This work was carried out during the tenure of an ERCIM "Alain Bensoussan" Fellowship Programme. The research leading to these results has received funding from the European Union Seventh Framework Programme (FP7/2007-2013) under grant agreement no 246016. 
contingency tables and to study relations among conditional probabilities, see [6] and [9. In general, it is not easy to describe the set $U(I)$ or even to estimate the degree of its elements. The case that $I$ is a toric ideal has been of particular interest, see for example [2] and [12.

In this article we study the universal Gröbner basis of $P_{G}$. Since the ideal $P_{G}$ is prime, it is also a toric ideal and therefore one can associate an integer program to it. The maximum degree of the elements in the universal Gröbner basis of $P_{G}$ is an important measure for the complexity of the aforementioned integer program. It is worth to note that there exists an upper bound on the degrees of the elements of $U\left(P_{G}\right)$ (see Theorem 4.7 in [1] ), however it is very far from being sharp.

In section 2 we show that if the graph $G$ is bipartite, then every initial ideal of $P_{G}$ is generated by squarefree monomials and also the universal Gröbner basis consists only of the circuits of $P_{G}$. For a bipartite graph $G$, we additionally prove that the maximum degree of a binomial in the universal Gröbner basis of $P_{G}$ is bounded above by $\left\lfloor\frac{m+n+1}{2}\right\rfloor$. Our bound is sharp, see Remark 3.13 and Remark 3.18 ,

An interesting problem is to determine when an ideal generated by binomials is the toric ideal $I_{H}$ associated to a finite graph $H$. In 8 H. Ohsugi and T. Hibi consider it for the class of ideals generated by adjacent 2-minors. In section 3 we study the above problem for ideals generated by diagonal 2-minors. More precisely, Theorem 3.8 asserts that, given a connected graph $G$, there exists a finite simple graph $H$ such that $P_{G}=I_{H}$ if and only if $G$ has at most one cycle. Our approach produces new examples of non-bipartite graphs $H$ such that the toric ideal $I_{H}$ has a quadratic Gröbner basis. Moreover, using Theorem 3.8 and Theorem 3.4 in [12, we characterize in graph theoretical terms the elements of the universal Gröbner basis of $P_{G}$, under the assumption that $G$ is a connected graph with at most one cycle.

\section{General Results for ideals generated By Diagonal 2-Minors}

In this section first we recall some basic facts about toric ideals associated to vector configurations. Next we associate to a simple graph $G$ on the vertex set $\{1, \ldots, n\}$ the vector configuration $A_{G}$, and let $N_{G}$ be the matrix with columns the vectors of $A_{G}$. It turns out (see Proposition 2.3) that $P_{G}$ is the toric ideal $I_{A_{G}}$ associated to $A_{G}$. Also, we show that the matrix $N_{G}$ is totally unimodular if and only if the graph $G$ is bipartite. As a consequence, if $G$ is a bipartite graph, then every initial ideal of $P_{G}$ is generated by squarefree monomials. Moreover, for a bipartite graph $G$, the universal Gröbner basis of $P_{G}$ is described by the circuits of $I_{A_{G}}$.

\subsection{Basics on toric ideals.}

Let $A=\left\{\mathbf{a}_{1}, \ldots, \mathbf{a}_{s}\right\} \subset \mathbb{Z}^{r}$ be a vector configuration and let $K\left[y_{1}, \ldots, y_{s}\right]$ be the polynomial ring in $s$ variables over a field $K$. As usual, we will denote by $\mathbf{y}^{\mathbf{u}}$ the monomial $y_{1}^{u_{1}} \cdots y_{s}^{u_{s}}$ of $K\left[y_{1}, \ldots, y_{s}\right]$, with $\mathbf{u}=\left(u_{1}, \ldots, u_{s}\right) \in \mathbb{N}^{s}$. The toric ideal $I_{A}$ is the kernel of the $K$-algebra homomorphism $\phi: K\left[y_{1}, \ldots, y_{s}\right] \rightarrow K\left[t_{1}^{ \pm 1}, \ldots, t_{r}^{ \pm 1}\right]$ given by

$$
\phi\left(y_{i}\right)=\mathbf{t}^{\mathbf{a}_{i}}=t_{1}^{a_{i, 1}} \cdots t_{r}^{a_{i, r}} \text { for all } i=1, \ldots, s,
$$

where $\mathbf{a}_{i}=\left(a_{i, 1}, \ldots, a_{i, r}\right)$. Thus every toric ideal is prime. Furthermore, the ideal $I_{A}$ is generated by all the binomials $\mathbf{y}^{\mathbf{u}}-\mathbf{y}^{\mathbf{v}}$ such that $\phi\left(\mathbf{y}^{\mathbf{u}}\right)=\phi\left(\mathbf{y}^{\mathbf{v}}\right)$, see [11]. 
Let $N=\left(a_{i, j}\right)$ be the $r \times s$ matrix with columns the vectors $\mathbf{a}_{1}, \ldots, \mathbf{a}_{s}$. By Lemma 4.2 in [11, the height of $I_{A}$, denoted by ht $\left(I_{A}\right)$, is equal to $s-g$, where $g$ is the rank of the matrix $N$.

For a vector $\mathbf{u}=\left(u_{1}, \ldots, u_{s}\right) \in \mathbb{Z}^{s}$, we let $\operatorname{supp}(\mathbf{u})=\left\{i \in\{1, \ldots, s\} \mid u_{i} \neq 0\right\}$ be the support of $\mathbf{u}$. The support of a binomial $B=\mathbf{y}^{\mathbf{u}}-\mathbf{y}^{\mathbf{v}}$ is $\operatorname{supp}(B)=$ $\operatorname{supp}(\mathbf{u}) \cup \operatorname{supp}(\mathbf{v})$. An irreducible binomial $B$ belonging to $I_{A}$ is called a circuit of $I_{A}$ if there exists no binomial $B^{\prime} \in I_{A}$ such that $\operatorname{supp}\left(B^{\prime}\right) \varsubsetneqq \operatorname{supp}(B)$. We shall denote by $\mathcal{C}\left(I_{A}\right)$ the set of circuits of $I_{A}$. The set $\mathcal{C}\left(I_{A}\right)$ can be computed easily, see [3] or [4. An irreducible binomial $\mathbf{y}^{\mathbf{u}}-\mathbf{y}^{\mathbf{v}}$ belonging to $I_{A}$ is called primitive if there is no other binomial $\mathbf{y}^{\mathbf{c}}-\mathbf{y}^{\mathbf{d}} \in I_{A}$ such that $\mathbf{y}^{\mathbf{c}}$ divides $\mathbf{y}^{\mathbf{u}}$ and $\mathbf{y}^{\mathbf{d}}$ divides $\mathbf{y}^{\mathbf{v}}$. The set of all primitive binomials is the Graver basis of $I_{A}$, denoted by $\operatorname{Gr}\left(I_{A}\right)$. For any toric ideal $I_{A}$, we have that $\mathcal{C}\left(I_{A}\right) \subseteq U\left(I_{A}\right) \subseteq \operatorname{Gr}\left(I_{A}\right)$, see [11.

Toric ideals associated to graphs serve as interesting examples of toric ideals. Let $H$ be a finite simple graph with vertices $V(H)=\left\{v_{1}, \ldots, v_{r}\right\}$ and edges $E(H)=$ $\left\{z_{1}, \ldots, z_{s}\right\}$. The incidence matrix of $H$ is the $r \times s$ matrix $M_{H}=\left(b_{i, j}\right)$ defined by

$$
b_{i, j}= \begin{cases}1, & \text { if } v_{i} \text { is one of the vertices in } z_{j} \\ 0, & \text { otherwise. }\end{cases}
$$

Let $\mathcal{B}_{H}=\left\{\mathbf{b}_{1}, \ldots, \mathbf{b}_{s}\right\}$ be the set of vectors in $\mathbb{Z}^{r}$, where $\mathbf{b}_{i}=\left(b_{i, 1}, \ldots, b_{i, r}\right)$ for $1 \leq i \leq s$. With $I_{H}$ we denote the toric ideal $I_{\mathcal{B}_{H}}$ in $K\left[y_{1}, \ldots, y_{s}\right]$. By Lemma 8.3.2 in [13, the rank of the matrix $M_{H}$ equals $r-c(H)$, where $c(H)$ is the number of connected components of $H$ which are bipartite. Thus the height of $I_{H}$ equals $s-r+c(H)$.

A walk of length $l$ of $H$ connecting $v_{i_{1}} \in V(H)$ with $v_{i_{l+1}} \in V(H)$ is a finite sequence of the form

$$
\Gamma=\left(\left\{v_{i_{1}}, v_{i_{2}}\right\},\left\{v_{i_{2}}, v_{i_{3}}\right\}, \ldots,\left\{v_{i_{l}}, v_{i_{l+1}}\right\}\right)
$$

with each $z_{i_{j}}=\left\{v_{i_{j}}, v_{i_{j+1}}\right\} \in E(H), 1 \leq j \leq l$. In some cases we may also denote a walk only by vertices $\left(v_{i_{1}}, v_{i_{2}}, \ldots, v_{i_{l}}, v_{i_{l+1}}\right)$. An even (respectively odd) walk is a walk of even (respectively odd) length. The walk $\Gamma$ is closed if $v_{i_{1}}=v_{i_{l+1}}$. A cycle is a closed walk $\left(\left\{v_{i_{1}}, v_{i_{2}}\right\},\left\{v_{i_{2}}, v_{i_{3}}\right\}, \ldots,\left\{v_{i_{l}}, v_{i_{1}}\right\}\right)$ with $l \geq 3$ and $v_{i_{j}} \neq v_{i_{k}}$, for every $1 \leq j<k \leq l$.

Given an even closed walk $\Gamma=\left(z_{i_{1}}, \ldots, z_{i_{2 l}}\right)$ of $H$, we denote by $B_{\Gamma}$ the binomial

$$
B_{\Gamma}:=\prod_{j=1}^{l} y_{i_{2 j-1}}-\prod_{j=1}^{l} y_{i_{2 j}} \in I_{H} .
$$

Moreover, Proposition 8.1.2 in 13 guarantees that $I_{H}$ is generated by all the binomials $B_{\Gamma}$, where $\Gamma$ is an even closed walk of $H$.

\subsection{Ideals generated by diagonal 2-minors.}

Let $G$ be a simple graph on the vertex set $\{1, \ldots, n\}$ with the edge set $E(G)=$ $\left\{z_{1}, \ldots, z_{m}\right\}$. Let $\left\{\mathbf{e}_{1}, \ldots, \mathbf{e}_{n}, \mathbf{e}_{n+1}, \ldots, \mathbf{e}_{n+m}\right\}$ be the canonical basis of $\mathbb{Z}^{n+m}$. Given an edge $z_{i}=\left\{i_{k}, i_{l}\right\}$ of $G$, we consider the following two vectors: $\mathbf{a}_{\left\{i_{k}, i_{l}\right\}}:=$ $\mathbf{e}_{i_{k}}+\mathbf{e}_{i_{l}}-\mathbf{e}_{n+i}$ and $\mathbf{b}_{\left\{i_{k}, i_{l}\right\}}:=\mathbf{e}_{n+i}$. Also, for every $1 \leq j \leq n$, we consider the vector $\mathbf{a}_{j j}:=\mathbf{e}_{j}$. Let $A_{G}$ denote the set

$$
A_{G}:=\left\{\mathbf{a}_{\{i, j\}}, \mathbf{b}_{\{i, j\}} \mid\{i, j\} \text { is an edge of } G\right\} \cup\left\{\mathbf{a}_{j j} \mid 1 \leq j \leq n\right\}
$$


consisting of $2 m+n$ vectors. Let $N_{G}$ be the $(n+m) \times(2 m+n)$ matrix whose columns are the vectors in $A_{G}$.

Example 2.1. Let $G$ be the graph on the vertex set $\{1, \ldots, 5\}$ with edges $z_{1}=$ $\{1,2\}, z_{2}=\{2,3\}, z_{3}=\{3,4\}, z_{4}=\{1,4\}, z_{5}=\{1,5\}$ and $z_{6}=\{3,5\}$. The set $A_{G}$ consists of the following 17 vectors: $\mathbf{a}_{\{1,2\}}=\mathbf{e}_{1}+\mathbf{e}_{2}-\mathbf{e}_{6}, \mathbf{b}_{\{1,2\}}=\mathbf{e}_{6}, \mathbf{a}_{\{2,3\}}=$ $\mathbf{e}_{2}+\mathbf{e}_{3}-\mathbf{e}_{7}, \mathbf{b}_{\{2,3\}}=\mathbf{e}_{7}, \mathbf{a}_{\{3,4\}}=\mathbf{e}_{3}+\mathbf{e}_{4}-\mathbf{e}_{8}, \mathbf{b}_{\{3,4\}}=\mathbf{e}_{8}, \mathbf{a}_{\{1,4\}}=\mathbf{e}_{1}+\mathbf{e}_{4}-\mathbf{e}_{9}$, $\mathbf{b}_{\{1,4\}}=\mathbf{e}_{9}, \mathbf{a}_{\{1,5\}}=\mathbf{e}_{1}+\mathbf{e}_{5}-\mathbf{e}_{10}, \mathbf{b}_{\{1,5\}}=\mathbf{e}_{10}, \mathbf{a}_{\{3,5\}}=\mathbf{e}_{3}+\mathbf{e}_{5}-\mathbf{e}_{11}, \mathbf{b}_{\{3,5\}}=\mathbf{e}_{11}$, $\mathbf{a}_{11}=\mathbf{e}_{1}, \mathbf{a}_{22}=\mathbf{e}_{2}, \mathbf{a}_{33}=\mathbf{e}_{3}, \mathbf{a}_{44}=\mathbf{e}_{4}, \mathbf{a}_{55}=\mathbf{e}_{5}$.

The toric ideal $I_{A_{G}}$ is the kernel of the $K$-algebra homomorphism

$$
\phi: S \rightarrow K\left[t_{1}^{ \pm 1}, \ldots, t_{11}^{ \pm 1}\right]
$$

given by $\phi\left(x_{12}\right)=t_{1} t_{2} t_{6}^{-1}, \phi\left(x_{21}\right)=t_{6}, \phi\left(x_{23}\right)=t_{2} t_{3} t_{7}^{-1}, \phi\left(x_{32}\right)=t_{7}, \phi\left(x_{34}\right)=$ $t_{3} t_{4} t_{8}^{-1}, \phi\left(x_{43}\right)=t_{8}, \phi\left(x_{14}\right)=t_{1} t_{4} t_{9}^{-1}, \phi\left(x_{41}\right)=t_{9}, \phi\left(x_{15}\right)=t_{1} t_{5} t_{10}^{-1}, \phi\left(x_{51}\right)=t_{10}$, $\phi\left(x_{35}\right)=t_{3} t_{5} t_{11}^{-1}, \phi\left(x_{53}\right)=t_{11}, \phi\left(x_{11}\right)=t_{1}, \phi\left(x_{22}\right)=t_{2}, \phi\left(x_{33}\right)=t_{3}, \phi\left(x_{44}\right)=t_{4}$, $\phi\left(x_{55}\right)=t_{5}$. Actually $I_{A_{G}}$ is generated by the following 6 binomials: $f_{12}, f_{23}, f_{34}$, $f_{14}, f_{15}$ and $f_{35}$. Thus $P_{G}=I_{A_{G}}$.

Given a subgraph $W$ of $G$, we let $P_{W}$ be the ideal of the polynomial ring

$$
K\left[\left\{x_{i j}, x_{j i} \mid\{i, j\} \in E(W)\right\} \cup\left\{x_{i i}\right\}_{1 \leq i \leq n}\right]
$$

generated by all the binomials $f_{i j}$, where $\{i, j\}$ is an edge of $W$.

Proposition 2.2. (5]) The ideal $P_{W}$ is complete intersection of height $|E(W)|$.

Proposition 2.3. Let $G$ be a simple graph on the vertex set $\{1, \ldots, n\}$ with $m$ edges, then the ideal $P_{G}$ coincides with the toric ideal $I_{A_{G}}$.

Proof. Given an edge $z_{i}=\left\{i_{k}, i_{l}\right\}$ of $G$, we have that

$$
\mathbf{a}_{\left\{i_{k}, i_{l}\right\}}+\mathbf{b}_{\left\{i_{k}, i_{l}\right\}}=\left(\mathbf{e}_{i_{k}}+\mathbf{e}_{i_{l}}-\mathbf{e}_{n+i}\right)+\mathbf{e}_{n+i}=\mathbf{e}_{i_{k}}+\mathbf{e}_{i_{l}}=\mathbf{a}_{i_{k} i_{k}}+\mathbf{a}_{i_{l} i_{l}},
$$

so the binomial $f_{i_{k} i_{l}}$ belongs to $I_{A_{G}}$, and therefore $P_{G} \subseteq I_{A_{G}}$. Moreover the rank of $N_{G}$ is equal to $n+m$, thus ht $\left(I_{A_{G}}\right)=2 m+n-(n+m)=m$. From Proposition 2.2 we have that $P_{G}$ is a prime ideal of height $m$. Thus $P_{G}=I_{A_{G}}$.

Remark 2.4. There is a term order such that the initial ideal of $I_{A_{G}}$ is generated by squarefree quadratic monomials. By Corollary 8.9 in [11, the vector configuration $A_{G}$ has a unimodular regular triangulation.

A matrix $M$ with $\operatorname{rank}(M)=d$ is called unimodular if all nonzero $d \times d$-minors of $M$ have the same absolute value. The matrix $M$ is called totally unimodular when every minor of $M$ is 0 or \pm 1 . It is well known (see for example [10, Chapter 19]) that the graph $G$ is bipartite if and only if its incidence matrix $M_{G}$ is totally unimodular.

Theorem 2.5. The matrix $N_{G}$ is totally unimodular if and only if the graph $G$ is bipartite.

Proof. $(\Rightarrow)$ Suppose that $N_{G}$ is totally unimodular. Then also $M_{G}$ is totally unimodular since it is a submatrix of $N_{G}$, and therefore $G$ is bipartite.

$(\Leftarrow)$ Suppose that $G$ is bipartite. Since total unimodularity is preserved under the unit vectors, it is enough to consider the matrix $Q$ with columns the vectors $\mathbf{a}_{\left\{i_{k}, i_{l}\right\}}=\mathbf{e}_{i_{k}}+\mathbf{e}_{i_{l}}-\mathbf{e}_{n+i}$, where $z_{i}=\left\{i_{k}, i_{l}\right\}$ is an edge of $G$. It suffices to prove that $Q$ is totally unimodular. The incidence matrix $M_{G}$ is totally unimodular, since 
$G$ is bipartite, so also the transpose $M_{G}^{T}$ of $M_{G}$ is totally unimodular. Thus the $m \times(n+m)$ matrix $V=\left(M_{G}^{T} \mid R\right)$, where $R$ is the matrix with rows $-\mathbf{e}_{1}, \ldots,-\mathbf{e}_{m}$, is totally unimodular, and therefore $Q=V^{T}$ is totally unimodular.

Theorem 2.6. Let $G$ be a simple bipartite graph on the vertex set $\{1, \ldots, n\}$, then the initial ideal of $P_{G}$ with respect to any term order is generated by squarefree monomials. Furthermore, the equality $U\left(P_{G}\right)=\mathcal{C}\left(I_{A_{G}}\right)$ holds.

Proof. We have, from Theorem 2.5. that the matrix $N_{G}$ is totally unimodular, and hence also unimodular. By Corollary 8.9 in [11, every initial ideal of $I_{A_{G}}$ is generated by squarefree monomials. Now, Proposition 8.11 in [11] asserts that $\mathcal{C}\left(I_{A_{G}}\right)=\operatorname{Gr}\left(I_{A_{G}}\right)$. Thus $U\left(I_{A_{G}}\right)=\mathcal{C}\left(I_{A_{G}}\right)$, and therefore $U\left(P_{G}\right)=\mathcal{C}\left(I_{A_{G}}\right)$ since $P_{G}=I_{A_{G}}$.

Remark 2.7. Let $G$ be a simple bipartite graph on the vertex set $\{1, \ldots, n\}$, then the matrix $N_{G}$ is unimodular. For every circuit $B=\mathbf{x}^{\mathbf{u}}-\mathbf{x}^{\mathbf{v}} \in I_{A_{G}}$ we have, from the proof of Proposition 8.11 in 11, that both $\mathbf{x}^{\mathbf{u}}$ and $\mathbf{x}^{\mathbf{v}}$ are squarefree monomials.

Corollary 2.8. Let $G$ be a simple bipartite graph on the vertex set $\{1, \ldots, n\}$ with $m$ edges, then the maximum degree of a binomial in the universal Gröbner basis of $P_{G}$ is less than or equal to $\left\lfloor\frac{m+n+1}{2}\right\rfloor$.

Proof. Given a circuit $B \in I_{A_{G}}$, we have, from Lemma 4.8 in [1], that $\operatorname{supp}(B)$ has at most $m+n+1$ elements. Since every binomial in $I_{A_{G}}$ is homogeneous with respect to the standard grading, we have that the degree of $B$ is less than or equal to $\left\lfloor\frac{m+n+1}{2}\right\rfloor$. By Theorem 2.6 the maximum degree of a binomial in the universal Gröbner basis of $P_{G}$ is less than or equal to $\left\lfloor\frac{m+n+1}{2}\right\rfloor$.

Example 2.9. We come back to Example 2.1 The circuits of $I_{A_{G}}$ are the following:

$$
\begin{aligned}
& \mathcal{C}\left(I_{A_{G}}\right)=\left\{f_{12}, f_{23}, f_{34}, f_{14}, f_{15}, f_{35}, x_{44} x_{35} x_{53}-x_{55} x_{34} x_{43},\right. \\
& x_{11} x_{35} x_{53}-x_{33} x_{15} x_{51}, x_{44} x_{15} x_{51}-x_{55} x_{14} x_{41}, x_{22} x_{15} x_{51}-x_{55} x_{12} x_{21}, \\
& x_{33} x_{14} x_{41}-x_{11} x_{34} x_{43}, x_{22} x_{14} x_{41}-x_{44} x_{12} x_{21}, x_{22} x_{34} x_{43}-x_{44} x_{23} x_{32}, \\
& x_{11} x_{23} x_{32}-x_{33} x_{12} x_{21}, x_{22} x_{35} x_{53}-x_{55} x_{23} x_{32}, x_{14} x_{41} x_{35} x_{53}-x_{34} x_{43} x_{15} x_{51} \text {, } \\
& x_{14} x_{41} x_{35} x_{53}-x_{33} x_{44} x_{15} x_{51}, x_{14} x_{41} x_{35} x_{53}-x_{11} x_{55} x_{34} x_{43}, \\
& x_{11} x_{44} x_{35} x_{53}-x_{34} x_{43} x_{15} x_{51}, x_{12} x_{21} x_{35} x_{53}-x_{23} x_{32} x_{15} x_{51} \text {, } \\
& x_{11} x_{22} x_{35} x_{53}-x_{23} x_{32} x_{15} x_{51}, x_{12} x_{21} x_{35} x_{53}-x_{22} x_{33} x_{15} x_{51}, \\
& x_{12} x_{21} x_{35} x_{53}-x_{11} x_{55} x_{23} x_{32}, x_{34} x_{43} x_{15} x_{51}-x_{33} x_{55} x_{14} x_{41}, \\
& x_{23} x_{32} x_{15} x_{51}-x_{33} x_{55} x_{12} x_{21}, x_{23} x_{32} x_{14} x_{41}-x_{12} x_{21} x_{34} x_{43}, \\
& x_{23} x_{32} x_{14} x_{41}-x_{11} x_{22} x_{34} x_{43}, x_{22} x_{33} x_{14} x_{41}-x_{12} x_{21} x_{34} x_{43}, \\
& x_{23} x_{32} x_{14} x_{41}-x_{33} x_{44} x_{12} x_{21}, x_{12} x_{21} x_{34} x_{43}-x_{11} x_{44} x_{23} x_{32}, \\
& x_{22} x_{14} x_{41} x_{35} x_{53}-x_{44} x_{23} x_{32} x_{15} x_{51}, x_{22} x_{14} x_{41} x_{35} x_{53}-x_{55} x_{12} x_{21} x_{34} x_{43}, \\
& x_{44} x_{12} x_{21} x_{35} x_{53}-x_{55} x_{23} x_{32} x_{14} x_{41}, x_{44} x_{12} x_{21} x_{35} x_{53}-x_{22} x_{34} x_{43} x_{15} x_{51} \text {, } \\
& \left.x_{22} x_{34} x_{43} x_{15} x_{51}-x_{55} x_{23} x_{32} x_{14} x_{41}, x_{44} x_{23} x_{32} x_{15} x_{51}-x_{55} x_{12} x_{21} x_{34} x_{43}\right\} \text {. }
\end{aligned}
$$

Notice that $G$ is a bipartite graph which has more than one cycles. By Theorem 2.6, the universal Gröbner basis of $P_{G}$ consists of the above 36 binomials. 


\section{Classification of all graphs $G$ such that the equality $P_{G}=I_{H}$ HOLDS}

Let $G$ be a simple graph on the vertex set $\{1, \ldots, n\}$. The next proposition gives a necessary condition for the equality $P_{G}=I_{H}$, where $H$ is a finite simple graph.

Proposition 3.1. Let $G$ be a connected simple graph on the vertex set $\{1, \ldots, n\}$ with $m$ edges. If there exists a finite simple graph $H$ such that $P_{G}=I_{H}$, then $G$ has at most one cycle.

Proof. Without loss of generality we can assume that $H$ has exactly $2 m+n$ edges and also that $H$ contains no isolated vertices. Recall that $P_{G}=I_{A_{G}}$, where $A_{G}=\left\{\mathbf{a}_{\{i, j\}}, \mathbf{b}_{\{i, j\}} \mid\{i, j\} \in E(G)\right\} \cup\left\{\mathbf{a}_{j j} \mid 1 \leq j \leq n\right\}$. Let

$$
\mathcal{B}_{H}=\left\{\mathbf{b}_{i j}, \mathbf{b}_{j i} \mid\{i, j\} \in E(G)\right\} \cup\left\{\mathbf{b}_{11}, \ldots, \mathbf{b}_{n n}\right\}
$$

be the set of columns of the incidence matrix $M_{H}$ of $H$. Every column of $M_{H}$ has exactly two nonzero entries and both of them are equal to 1 . In particular, every $\mathbf{b}_{i i}, 1 \leq i \leq n$, has exactly two nonzero entries, so the cardinality of the set $\left\{\operatorname{supp}\left(\mathbf{b}_{11}\right), \ldots, \operatorname{supp}\left(\mathbf{b}_{n n}\right)\right\}$ is at most $2 n$. Given an edge $\{i, j\}$ of $G$, we have that $f_{i j}=x_{i i} x_{j j}-x_{i j} x_{j i} \in P_{G}$, so $f_{i j} \in I_{H}$ and therefore $\mathbf{b}_{i j}=\mathbf{b}_{i i}+\mathbf{b}_{j j}-\mathbf{b}_{j i}$. If $\operatorname{supp}\left(\mathbf{b}_{j i}\right) \cap \operatorname{supp}\left(\mathbf{b}_{i i}\right)=\emptyset$ or $\operatorname{supp}\left(\mathbf{b}_{j i}\right) \cap \operatorname{supp}\left(\mathbf{b}_{j j}\right)=\emptyset$, then the vector $\mathbf{b}_{i j}$ has at least one entry which is equal to -1 , a contradiction. Thus the cardinality of the set

$$
\mathcal{R}=\left\{\operatorname{supp}\left(\mathbf{b}_{i j}\right), \operatorname{supp}\left(\mathbf{b}_{j i}\right) \mid\{i, j\} \in E(G)\right\} \cup\left\{\operatorname{supp}\left(\mathbf{b}_{11}\right), \ldots, \operatorname{supp}\left(\mathbf{b}_{n n}\right)\right\}
$$

is at most $2 n$. Let $d$ be the number of vertices of $H$, then $d$ is less than or equal to $2 n$. Let $c(H)$ be the number of connected components of $H$ which are bipartite. By Proposition 2.2 we have that $\mathrm{ht}\left(P_{G}\right)=m$. Using the equality $\operatorname{ht}\left(P_{G}\right)=\operatorname{ht}\left(I_{H}\right)$, we deduce that $m=2 m+n-d+c(H)$, and therefore $m+n+c(H)=d \leq 2 n$. So $m \leq m+c(H) \leq n$, while $n \leq m+1$ since $G$ is connected. Thus $n \in\{m, m+1\}$, and therefore $G$ has at most one cycle. Note that $G$ is a tree when $n=m+1$, while $G$ has exactly one cycle in the case that $n=m$.

Let $W$ be a connected simple graph with $k$ vertices and $l$ edges. Consider two simple graphs $W_{1}, W_{2}$ with $V\left(W_{1}\right)=\left\{p_{1}, \ldots, p_{k}\right\}$ and $V\left(W_{2}\right)=\left\{q_{1}, \ldots, q_{k}\right\}$ such that $V\left(W_{1}\right) \cap V\left(W_{2}\right)=\emptyset$. Suppose that both $W_{1}$ and $W_{2}$ are isomorphic to $W$. More precisely, we assume that $\tau: V(W) \longrightarrow V\left(W_{1}\right), i \mapsto p_{i}$, is a bijection such that

$$
\{i, j\} \in E(W) \Longleftrightarrow\left\{p_{i}, p_{j}\right\} \in E\left(W_{1}\right),
$$

and also $\psi: V(W) \longrightarrow V\left(W_{2}\right), i \mapsto q_{i}$, is a bijection such that

$$
\{i, j\} \in E(W) \Longleftrightarrow\left\{q_{i}, q_{j}\right\} \in E\left(W_{2}\right) \text {. }
$$

We will define a new graph $W^{\star}$ with $2 k$ vertices and $k+2 l$ edges as follows. The vertex set of $W^{\star}$ is $V\left(W_{1}\right) \cup V\left(W_{2}\right)$. Also both $W_{1}$ and $W_{2}$ are subgraphs of $W^{\star}$, so each one of the edges of $W_{1}, W_{2}$ is also an edge of $W^{\star}$. Finally, for every vertex $i$ of $W$, we let $\left\{p_{i}, q_{i}\right\}$ be an edge of $W^{\star}$. Note that $W^{\star}$ is a connected graph. The graph $W^{\star}$ is commonly known as a prism of $W$.

Remark 3.2. Let $G$ be a simple graph on the vertex set $\{1, \ldots, n\}$ and let $W$ be a connected subgraph of $G$. Given an edge $\{i, j\}$ of $W$, we have that

$$
\Gamma=\left(d_{i i}=\left\{p_{i}, q_{i}\right\}, d_{j i}=\left\{q_{i}, q_{j}\right\}, d_{j j}=\left\{p_{j}, q_{j}\right\}, d_{i j}=\left\{p_{i}, p_{j}\right\}\right)
$$


is an even cycle of $W^{\star}$, and therefore $x_{i i} x_{j j}-x_{i j} x_{j i}=B_{\Gamma} \in I_{W^{\star}}$. Thus $P_{W} \subseteq I_{W^{\star}}$.

Lemma 3.3. Let $G$ be a simple graph on the vertex set $\{1, \ldots, n\}$ and $W$ be a connected subgraph of $G$. If $W$ is either a tree or a non-bipartite graph with exactly one cycle, then $P_{W}=I_{W^{\star}}$ where $W^{\star}$ is the graph constructed above.

Proof. Let $W$ be a tree with $k$ vertices and $l=k-1$ edges. By Proposition 2.2 we have that $h t\left(P_{W}\right)=l$. If the graph $W^{\star}$ is not bipartite, then

$$
\operatorname{ht}\left(I_{W^{\star}}\right)=(k+2 l)-2 k=2 l-k=2 l-(l+1)=l-1<l,
$$

and therefore ht $\left(I_{W^{\star}}\right)<\operatorname{ht}\left(P_{W}\right)$ a contradiction to the fact that $P_{W} \subseteq I_{W^{\star}}$. Thus $W^{\star}$ is bipartite, so $h t\left(I_{W^{\star}}\right)=(k+2 l)-2 k+1=l$, and therefore $P_{W}=I_{W^{\star}}$.

Let $W$ be a non-bipartite graph with exactly one cycle. Let $r$ and $s$ be the number of vertices and edges, respectively, of $W$, then $r=s$. We have that the graph $W^{\star}$ is not bipartite, and therefore

$$
\operatorname{ht}\left(I_{W^{\star}}\right)=(r+2 s)-2 r=2 s-r=s=\operatorname{ht}\left(P_{W}\right) .
$$

Since $P_{W} \subseteq I_{W^{\star}}$, we obtain the equality $P_{W}=I_{W^{\star}}$.

Remark 3.4. Given an even cycle $C=(\{1,2\},\{2,3\}, \ldots,\{k, 1\})$ of a simple graph $G$, we have that the graph $C^{\star}$ is bipartite, since

$$
\left\{p_{1}, p_{3}, p_{5}, \ldots, p_{k-1}, q_{2}, q_{4}, \ldots, q_{k}\right\} \cup\left\{p_{2}, p_{4}, \ldots, p_{k}, q_{1}, q_{3}, q_{5}, \ldots, q_{k-1}\right\}
$$

is a partition of its vertices. Thus $\mathrm{ht}\left(I_{C^{*}}\right)=3 k-2 k+1=k+1 \neq k=\operatorname{ht}\left(P_{C}\right)$, and therefore $P_{C} \neq I_{C^{\star}}$.

Let $C=\left(\left\{i_{1}, i_{2}\right\},\left\{i_{2}, i_{3}\right\}, \ldots,\left\{i_{k}, i_{1}\right\}\right)$ be an even cycle of a graph $G$ of length $k \geq 4$. Consider two simple graphs $C_{1}, C_{2}$ with $V\left(C_{1}\right)=\left\{v_{1}, \ldots, v_{k}\right\}$ and $V\left(C_{2}\right)=$ $\left\{w_{1}, \ldots, w_{k}\right\}$ such that $V\left(C_{1}\right) \cap V\left(C_{2}\right)=\emptyset$. Suppose that both $C_{1}$ and $C_{2}$ are isomorphic to the path $Y=\left(\left\{i_{1}, i_{2}\right\},\left\{i_{2}, i_{3}\right\}, \ldots,\left\{i_{k-1}, i_{k}\right\}\right)$. More precisely, we assume that $\tau: V(Y) \longrightarrow V\left(C_{1}\right), r \mapsto v_{r}$, is a bijection such that

$$
\{r, s\} \in E(Y) \Longleftrightarrow z_{r s}:=\left\{v_{r}, v_{s}\right\} \in E\left(C_{1}\right),
$$

and also $\psi: V(Y) \longrightarrow V\left(C_{2}\right), r \mapsto w_{r}$, is a bijection such that

$$
\{r, s\} \in E(Y) \Longleftrightarrow z_{s r}:=\left\{w_{r}, w_{s}\right\} \in E\left(C_{2}\right) .
$$

We will define a new graph $\bar{C}$ with $2 k$ vertices and $3 k$ edges as follows. The vertex set of $\bar{C}$ is $V\left(C_{1}\right) \cup V\left(C_{2}\right)$. Also both $C_{1}$ and $C_{2}$ are subgraphs of $\bar{C}$. Additionally, for $r=i_{1}$ and $s=i_{k}$, we let $z_{r s}:=\left\{v_{r}, w_{s}\right\}$ and $z_{s r}:=\left\{v_{s}, w_{r}\right\}$ be edges of $\bar{C}$. Finally, for every vertex $r$ of $C$, we let $z_{r r}:=\left\{v_{r}, w_{r}\right\}$ be an edge of $\bar{C}$. Note that $\bar{C}$ is a connected graph. The graph $\bar{C}$ is commonly known as a twisted prism of $C$. The next lemma asserts that $P_{C}=I_{\bar{C}}$.

Lemma 3.5. Let $G$ be a simple graph on the vertex set $\{1, \ldots, n\}$ and $C$ be an even cycle of $G$ of length $k \geq 4$. Then $P_{C}=I_{\bar{C}}$ for the graph $\bar{C}$ constructed above.

Proof. Let $C=\left(\left\{i_{1}, i_{2}\right\},\left\{i_{2}, i_{3}\right\}, \ldots,\left\{i_{k}, i_{1}\right\}\right)$, then the graph $\bar{C}$ is not bipartite since $\Gamma=\left(v_{i_{1}}, w_{i_{k}}, v_{i_{k}}, v_{i_{k-1}}, \ldots, v_{i_{2}}, v_{i_{1}}\right)$ is an odd cycle of length $k+1$. Thus $\operatorname{ht}\left(I_{\bar{C}}\right)=3 k-2 k=k$. For every edge $\{r, s\}$ of $Y$ we have that $\Gamma=\left(z_{r r}, z_{s r}, z_{s s}, z_{r s}\right)$ is an even cycle of $\bar{C}$, so $x_{r r} x_{s s}-x_{r s} x_{s r}=B_{\Gamma} \in I_{\bar{C}}$. Moreover, for $r=i_{1}$ and $s=i_{k}$, we have that also $\Gamma=\left(z_{r r}, z_{s r}, z_{s s}, z_{r s}\right)$ is an even cycle of $\bar{C}$, so $x_{r r} x_{s s}-x_{r s} x_{s r} \in I_{\bar{C}}$, and therefore $P_{C} \subseteq I_{\bar{C}}$. Thus the equality $P_{C}=I_{\bar{C}}$ holds, since, from Proposition 2.2, ht $\left(P_{C}\right)=k$. 
Remark 3.6. Given an even cycle $C=(\{1,2\},\{2,3\}, \ldots,\{k, 1\})$ of length $k \geq 4$ of $G$, we have that the graph $\bar{C}$ contains at least one even cycle of length $2 k$, namely

$$
\left(v_{1}, v_{2}, \ldots, v_{k-1}, v_{k}, w_{k}, w_{k-1}, \ldots, w_{1}, v_{1}\right) .
$$

Let $G_{1}=\left(V\left(G_{1}\right), E\left(G_{1}\right)\right), G_{2}=\left(V\left(G_{2}\right), E\left(G_{2}\right)\right)$ be graphs such that $G_{1} \cap G_{2}$ is a complete graph. The new graph $G=G_{1} \oplus G_{2}$ with the vertex set $V(G)=$ $V\left(G_{1}\right) \cup V\left(G_{2}\right)$ and edge set $E(G)=E\left(G_{1}\right) \cup E\left(G_{2}\right)$ is called the clique sum of $G_{1}$ and $G_{2}$ in $G_{1} \cap G_{2}$. If the cardinality of $V\left(G_{1}\right) \cap V\left(G_{2}\right)$ is $k+1$, then this operation is called a $k$-clique sum of the graphs $G_{1}$ and $G_{2}$. We write $G=G_{1} \bigoplus_{\widehat{v}} G_{2}$ to indicate that $G$ is the clique sum of $G_{1}$ and $G_{2}$ and that $V\left(G_{1}\right) \cap V\left(G_{2}\right)=\widehat{v}$.

Example 3.7. Let $G$ be the graph on the vertex set $\{1, \ldots, 6\}$ consisting of the even cycle $C=(\{1,2\},\{2,3\},\{3,4\},\{1,4\})$ and the tree $T$ with edges $\{1,5\}$ and $\{1,6\}$. Let $\bar{C}$ be the graph on the vertex set $\left\{v_{1}=7, v_{2}=8, v_{3}=9, v_{4}=10\right\} \cup\left\{w_{1}=\right.$ $\left.12, w_{2}=13, w_{3}=15, w_{4}=17\right\}$ consisting of the edges $z_{12}=\{7,8\}, z_{23}=\{8,9\}$, $z_{34}=\{9,10\}, z_{14}=\{7,17\}, z_{21}=\{12,13\}, z_{32}=\{13,15\}, z_{43}=\{15,17\}, z_{41}=$ $\{10,12\}, z_{11}=\{7,12\}, z_{22}=\{8,13\}, z_{33}=\{9,15\}, z_{44}=\{10,17\}$.

Also consider the graph $T^{\star}$ on the vertex set $\left\{v_{1}=7, p_{5}=18, p_{6}=19\right\} \cup\left\{w_{1}=\right.$ $\left.12, q_{5}=20, q_{6}=21\right\}$ consisting of the edges $z_{15}=\{7,18\}, z_{16}=\{7,19\}, z_{51}=$ $\{12,20\}, z_{61}=\{12,21\}, z_{11}, z_{55}=\{18,20\}, z_{66}=\{19,21\}$. Notice that $\bar{C} \cap T^{\star}$ is the graph on the vertex set $\widehat{v}=\{7,12\}$ consisting of the edge $z_{11}$. The 1 -clique sum $H$ of the graphs $\bar{C}$ and $T^{\star}$ is drawn in Figure 1 .

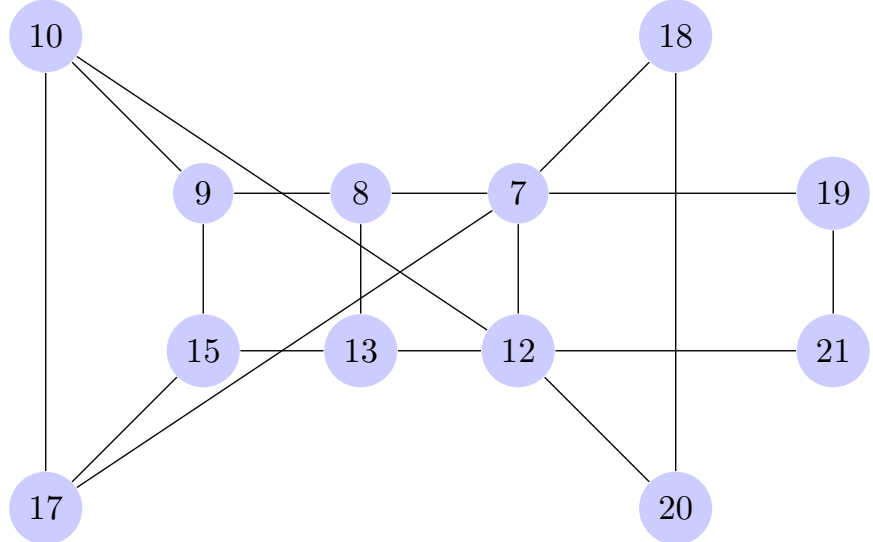

Figure 1.

It is easy to see that $P_{G} \subseteq I_{H}$. Moreover, $\operatorname{ht}\left(P_{G}\right)=6$, and also ht $\left(I_{H}\right)=18-12=6$ since the graph $H$ is not bipartite. Thus $P_{G}=I_{H}$, so $\left\{f_{12}, f_{23}, f_{34}, f_{14}, f_{15}, f_{16}\right\}$ is a quadratic Gröbner basis for $I_{H}$ with respect to the reverse lexicographic term order given by

$$
\begin{gathered}
x_{11}>x_{12}>x_{14}>x_{15}>x_{16}>x_{21}>x_{22}>x_{23}>x_{32}>x_{33}>x_{34}> \\
x_{41}>x_{43}>x_{44}>x_{51}>x_{55}>x_{61}>x_{66} .
\end{gathered}
$$

Let $W$ be a subgraph of $G$. Given an ideal $J$ in the polynomial ring

$$
K\left[\left\{x_{i j}, x_{j i} \mid\{i, j\} \in E(W)\right\} \cup\left\{x_{i i}\right\}_{1 \leq i \leq n}\right],
$$

we write $J^{e}$ for the ideal $J^{e}:=J \cdot S$.

The following theorem determines all connected graphs $G$ such that the ideal $P_{G}$ is of the form $I_{H}$, for a finite simple graph $H$. 
Theorem 3.8. Let $G$ be a connected simple graph on the vertex set $\{1, \ldots, n\}$. Then, there exists a finite simple graph $H$ such that $P_{G}=I_{H}$ if and only if $G$ has at most one cycle.

Proof. If there exists a finite simple graph $H$ such that $P_{G}=I_{H}$, then we have, from Proposition 3.1 that $G$ has at most one cycle. Conversely if either $G$ has no cycle or $G$ is non-bipartite with exactly one cycle, then Lemma 3.3 asserts that $P_{G}=I_{G^{\star}}$. Thus it is enough to consider the case that $G$ is bipartite with exactly one cycle. Let $C$ be the unique cycle of $G$ and suppose that it has length $k \geq 4$, where $k$ is even. The graph $G$ can be written as the 0 -clique sum of the cycle $C$ and some trees. More precisely, we have that

$$
G=C \bigoplus_{i_{1}} T_{1} \bigoplus_{i_{2}} T_{2} \bigoplus_{i_{3}} \cdots \bigoplus_{i_{s}} T_{s}
$$

for some vertices $i_{1}, \ldots, i_{s}$ of $C$. By Lemma 3.5, there is a connected graph $\bar{C}$ such that $P_{C}=I_{\bar{C}}$. Moreover, $\bar{C}$ has exactly $2 k$ vertices and $3 k$ edges. We shall denote by $\widehat{v_{j}}$ the set of vertices of the edge $z_{i_{j} i_{j}}, 1 \leq j \leq s$. By Lemma 3.3, for each $1 \leq j \leq s$ there exists a connected graph $T_{j}^{\star}$ such that $P_{T_{j}}=I_{T_{j}^{\star}}$. Without loss of generality we can assume that $V\left(T_{j}^{\star}\right) \cap V(\bar{C})=\widehat{v_{j}}$, for every $1 \leq j \leq s$, and also $V\left(T_{j}^{\star}\right) \cap V\left(T_{k}^{\star}\right)=\emptyset$, for every $j \neq k$. Let us suppose that the tree $T_{j}, 1 \leq j \leq s$, has $g_{j}$ edges, then $T_{j}^{\star}$ has $2 g_{j}+2$ vertices and $3 g_{j}+1$ edges. Let

$$
H=\bar{C} \bigoplus_{\widehat{v_{1}}} T_{1}^{\star} \bigoplus_{\widehat{v_{2}}} T_{2}^{\star} \bigoplus_{\widehat{v_{3}}} \cdots \bigoplus_{\widehat{v_{s}}} T_{s}^{\star}
$$

be the 1-clique sum of the graphs $\bar{C}$ and $T_{j}^{\star}, 1 \leq j \leq s$. We have that $P_{G}=$ $\left(P_{C}\right)^{e}+\left(P_{T_{1}}\right)^{e}+\cdots+\left(P_{T_{s}}\right)^{e}$, so $P_{G}=\left(I_{\bar{C}}\right)^{e}+\left(I_{T_{1}^{\star}}\right)^{e}+\cdots+\left(I_{T_{s}^{\star}}\right)^{e}$, and therefore $P_{G} \subseteq I_{H}$. Notice that, from Proposition $2.2 \mathrm{ht}\left(P_{G}\right)=k+g_{1}+\cdots+g_{s}$, and also

$$
\operatorname{ht}\left(I_{H}\right)=\left(3 k+3 g_{1}+\cdots+3 g_{s}\right)-\left(2 k+2 g_{1}+\cdots+2 g_{s}\right)=k+g_{1}+\cdots+g_{s} .
$$

Consequently $P_{G}=I_{H}$.

Remark 3.9. Let $G$ be a connected simple graph on the vertex set $\{1, \ldots, n\}$ with exactly one cycle. Let $W$ be either the graph $H$ constructed in the proof of Theorem 3.8 (when $G$ is bipartite) or the graph $G^{\star}$ when $G$ is non-bipartite. In both cases the graph $W$ is non-bipartite. The toric ideal $I_{W}$ has a quadratic Gröbner basis with respect to the reverse lexicographic term order on $S$ induced by the natural ordering of variables

$$
x_{11}>x_{12}>\cdots>x_{1 n}>x_{21}>x_{22}>\cdots>x_{2 n}>\cdots>x_{n 1}>\cdots>x_{n n} .
$$

Example 3.10. Consider the graph $G$ on the vertex set $\{1, \ldots, 4\}$ with edges $d_{12}=\{1,2\}, d_{23}=\{2,3\}, d_{13}=\{1,3\}$ and $d_{14}=\{1,4\}$. The graph $G$ has exactly one odd cycle, namely $\Gamma=\left(d_{12}, d_{23}, d_{13}\right)$. We let $G^{\star}$ be the graph with vertices $\{1,2,3,4\} \cup\{5,6,7,8\}$ and 12 edges, namely the 4 edges of $G$ and the edges $d_{21}=\{5,6\}, d_{32}=\{6,7\}, d_{31}=\{5,7\}, d_{41}=\{5,8\}, d_{11}=\{1,5\}, d_{22}=\{2,6\}$, $d_{33}=\{3,7\}, d_{44}=\{4,8\}$. By Lemma 3.3. the equality $P_{G}=I_{G^{*}}$ holds. Thus the set $\left\{f_{12}, f_{23}, f_{13}, f_{14}\right\}$ constitutes a quadratic Gröbner basis for the toric ideal $I_{G^{*}}$ with respect to the reverse lexicographic term order given by

$$
x_{11}>x_{12}>x_{13}>x_{14}>x_{21}>x_{22}>x_{23}>x_{31}>x_{32}>x_{33}>x_{41}>x_{44} .
$$


Using Algorithm 7.2 in [11, we determine the Graver basis of $I_{G^{\star}}$ which consists of the following 16 binomials:

$$
\begin{gathered}
f_{12}, f_{23}, f_{13}, f_{14}, x_{33} x_{14} x_{41}-x_{44} x_{13} x_{31}, x_{22} x_{14} x_{41}-x_{44} x_{12} x_{21}, \\
x_{22} x_{13} x_{31}-x_{11} x_{23} x_{32}, x_{22} x_{13} x_{31}-x_{33} x_{12} x_{21}, x_{11} x_{23} x_{32}-x_{33} x_{12} x_{21}, \\
x_{23} x_{32} x_{14} x_{41}-x_{22} x_{44} x_{13} x_{31}, x_{23} x_{32} x_{14} x_{41}-x_{33} x_{44} x_{12} x_{21}, x_{33}^{2} x_{12} x_{21}-x_{23} x_{32} x_{13} x_{31}, \\
x_{11}^{2} x_{23} x_{32}-x_{12} x_{21} x_{13} x_{31}, x_{22}^{2} x_{13} x_{31}-x_{12} x_{21} x_{23} x_{32}, \\
x_{11} x_{23} x_{32} x_{14} x_{41}-x_{44} x_{12} x_{21} x_{13} x_{31}, x_{12} x_{21} x_{13} x_{31} x_{44}^{2}-x_{23} x_{32} x_{14}^{2} x_{41}^{2} .
\end{gathered}
$$

Notice that $\operatorname{Gr}\left(I_{G^{\star}}\right) \neq \mathcal{C}\left(I_{G^{\star}}\right)$, since the binomial $B=x_{11} x_{23} x_{32} x_{14} x_{41}-x_{44} x_{12} x_{21} x_{13} x_{31}$ is primitive and not a circuit of $I_{G^{\star}}$. Thus $U\left(P_{G}\right) \neq \mathcal{C}\left(I_{G^{\star}}\right)$.

Let $G$ be a connected, simple and bipartite graph with exactly one cycle $C$. Consider the 1-clique sum $H$ of the graphs $\bar{C}$ and $T_{j}^{\star}, 1 \leq j \leq s$, which appeared in the proof of Theorem 3.8. Note that $I_{A_{G}}=I_{H}$, since $P_{G}=I_{H}$. By Theorem 2.6. the equality $U\left(P_{G}\right)=\mathcal{C}\left(I_{H}\right)$ holds. Theorem 3.12 describes all elements of $U\left(P_{G}\right)$. In order to prove this theorem, we will use the following result:

Theorem 3.11. (13]) Let $W$ be a finite, connected and simple graph. Then a binomial $B$ is a circuit of $I_{W}$ if and only if $B=B_{\Gamma}$ where $\Gamma$ is one of the following even closed walks of $W$ :

(1) $\Gamma$ is an even cycle.

(2) $\Gamma=\left(C_{1}, C_{2}\right)$, where $C_{1}$ and $C_{2}$ are odd cycles of $W$ having exactly one common vertex.

(3) $\Gamma=\left(C_{1}, e_{1}, \ldots, e_{r}, C_{2}, e_{r}, \ldots, e_{1}\right)$, where $C_{1}$ and $C_{2}$ are odd cycles of $W$ having no common vertex and where $\left(e_{1}, \ldots, e_{r}\right)$ is a path of $W$ which connects a vertex of $C_{1}$ and a vertex of $C_{2}$.

Theorem 3.12. Let $G$ be a connected simple graph on the vertex set $\{1, \ldots, n\}$. Suppose that $G$ is bipartite with exactly one cycle. Then the universal Gröbner basis of $P_{G}$ is given by the set

$$
U\left(P_{G}\right)=\left\{B_{\Gamma} \mid \Gamma \text { is an even cycle of } H\right\} .
$$

Proof. Let $C=(\{1,2\},\{2,3\}, \ldots,\{k, 1\})$ be the unique cycle of $G$, where $k \geq 4$ is even. Let $\left\{v_{1}, \ldots, v_{k}, w_{1}, \ldots, w_{k}\right\}$ be the set of vertices of $\bar{C}$, where $\left\{v_{1}, \ldots, v_{k}\right\} \cap$ $\left\{w_{1}, \ldots, w_{k}\right\}=\emptyset$. Every odd cycle in $H$ contains at least one of the edges $z_{1 k}=$ $\left\{v_{1}, w_{k}\right\}$ or $z_{k 1}=\left\{w_{1}, v_{k}\right\}$, since the subgraph $F$ of $H$ with the edge set $E(F)=$ $E(\bar{C}) \backslash\left\{z_{1 k}, z_{k 1}\right\}$ is bipartite and a partition of its vertices is

$$
\left\{v_{1}, v_{3}, \ldots, v_{k-1}, w_{2}, w_{4}, \ldots, w_{k}\right\} \cup\left\{v_{2}, v_{4}, \ldots, v_{k}, w_{1}, w_{3}, \ldots, w_{k-1}\right\} .
$$

We will prove that any two odd cycles in $H$ have at least 2 vertices in common. Suppose that there exist two odd cycles $\Gamma_{1}$ and $\Gamma_{2}$ in $H$ which share at most one vertex. Let, say, that $\Gamma_{1}$ contains $z_{1 k}$ and $\Gamma_{2}$ contains $z_{k 1}$. We can take the cycles $\Gamma_{1}$ and $\Gamma_{2}$ to start from the vertices $v_{1}$ and $w_{1}$, respectively. Moreover, we can assume that $z_{1 k}$ and $z_{k 1}$ are the first edges of $\Gamma_{1}$ and $\Gamma_{2}$, respectively. We claim that the second edge of $\Gamma_{1}$ is $\left\{w_{k}, w_{k-1}\right\}$. If $\left\{w_{k}, w_{k-1}\right\}$ is not the second edge, then either $\left\{w_{k}, v_{k}\right\}$ is the second edge of $\Gamma_{1}$ or there exists a vertex $q \in T_{i}^{\star}$ with $q \neq v_{k}$ such that $\left\{w_{k}, q\right\}$ is the second edge of $\Gamma_{1}$. In the latter case there exists a path in $\Gamma_{1}$ of length $>2$ connecting $w_{k}$ with $v_{k}$. Since $\Gamma_{1}$ is a cycle, we have that, in both cases, $\left\{v_{k}, v_{k-1}\right\}$ is an edge of $\Gamma_{1}$. Also $\left\{w_{1}, v_{k}\right\}$ is the first edge of $\Gamma_{2}$, so either $\left\{v_{k}, v_{k-1}\right\}$ is the second edge of $\Gamma_{2}$ or there exists a path in $\Gamma_{2}$ of length 
$\geq 1$ connecting $v_{k}$ with $w_{k}$. In both cases we arrive at a contradiction, since the cycles $\Gamma_{1}, \Gamma_{2}$ have at most one common vertex. Consequently, $\left\{w_{k}, w_{k-1}\right\}$ is the second edge of $\Gamma_{1}$ and analogously we have that $\left\{v_{k}, v_{k-1}\right\}$ is the second edge of $\Gamma_{2}$. Using similar arguments we conclude that $\left\{v_{1}, w_{k}\right\},\left\{w_{k}, w_{k-1}\right\}, \ldots,\left\{w_{3}, w_{2}\right\}$ are all edges of $\Gamma_{1}$, while $\left\{w_{1}, v_{k}\right\},\left\{v_{k}, v_{k-1}\right\}, \ldots,\left\{v_{3}, v_{2}\right\}$ are all edges of $\Gamma_{2}$. We claim that $\left\{w_{2}, w_{1}\right\}$ is also an edge of $\Gamma_{1}$. Suppose not, then either $\left\{w_{2}, v_{2}\right\}$ is an edge of $\Gamma_{1}$ or there exists a vertex $q^{\prime} \in T_{j}^{\star}$ with $q^{\prime} \neq w_{1}$ such that $\left\{w_{2}, q^{\prime}\right\}$ is an edge of $\Gamma_{1}$. In both cases there exists a path in $\Gamma_{1}$ of length $\geq 1$ connecting $w_{2}$ with $v_{2}$. Thus $\left\{v_{2}, v_{1}\right\}$ is an edge of $\Gamma_{1}$. But $\left\{v_{3}, v_{2}\right\}$ is an edge of $\Gamma_{2}$, so either $\left\{v_{2}, v_{1}\right\}$ is an edge of $\Gamma_{2}$ or there exists a path in $\Gamma_{2}$ of length $\geq 1$ connecting $v_{2}$ with $w_{2}$. Since the cycles $\Gamma_{1}, \Gamma_{2}$ have at most one vertex in common, we arrive at a contradiction. Thus $\left\{w_{2}, w_{1}\right\}$ is an edge of $\Gamma_{1}$ and analogously $\left\{v_{2}, v_{1}\right\}$ is an edge of $\Gamma_{2}$. But then $\Gamma_{1}, \Gamma_{2}$ have 2 vertices in common, namely $v_{1}$ and $w_{1}$, contradicting our assumption. As a consequence any two odd cycles in $H$ have at least 2 vertices in common. From Theorem 3.11 we have that every circuit of $I_{H}$ is of the form $B_{\Gamma}$, where $\Gamma$ is an even cycle of $H$. Consequently, the universal Gröbner basis of $P_{G}$ consists of all binomials of the form $B_{\Gamma}$, where $\Gamma$ is an even cycle of $H$.

Remark 3.13. Let $C=(\{1,2\},\{2,3\}, \ldots,\{k, 1\})$ be an even cycle of $G$ of length $k$, then $P_{C}=I_{\bar{C}}$, and therefore, from Remark 3.6. the maximum degree of a binomial in the universal Gröbner basis of $P_{C}$ is equal to $k$. Notice that $\left\lfloor\frac{k+k+1}{2}\right\rfloor=k$.

Proposition 3.14. Let $G$ be a simple graph on the vertex set $\{1, \ldots, n\}$ and $C=\left(\left\{p_{1}, p_{2}\right\},\left\{p_{2}, p_{3}\right\}, \ldots,\left\{p_{k}, p_{1}\right\}\right)$ be an odd cycle of $G$ of length $k \geq 3$. Consider the graph $C^{\star}$ on the vertex set $\left\{p_{1}, \ldots, p_{k}, q_{1}, \ldots, q_{k}\right\}$, where $\left\{p_{1}, \ldots, p_{k}\right\} \cap$ $\left\{q_{1}, \ldots, q_{k}\right\}=\emptyset$, and let $C^{\prime}$ be the odd cycle

$$
C^{\prime}=\left(\left\{q_{1}, q_{2}\right\},\left\{q_{2}, q_{3}\right\}, \ldots,\left\{q_{k-1}, q_{k}\right\},\left\{q_{k}, q_{1}\right\}\right) .
$$

Then a binomial $B \in P_{C}$ belongs to the universal Gröbner basis of $P_{C}$ if and only if $B=B_{\Gamma}$ where $\Gamma$ is one of the following even closed walks of $C^{\star}$ :

(1) $\Gamma$ is an even cycle of $C^{\star}$,

(2) $\Gamma=\left(C,\left\{p_{i}, q_{i}\right\}, C^{\prime}\right)$ where $1 \leq i \leq k$.

Proof. Since the equality $P_{C}=I_{C^{\star}}$ holds, we deduce from Proposition 2.2 that the toric ideal $I_{C^{\star}}$ is complete intersection. Thus, from Proposition 6.1 in [1], the graph $C^{\star}$ has exactly two vertex disjoint odd cycles, namely $C$ and $C^{\prime}$. By Lemma 3.2 in [7, every primitive binomial $B \in I_{C^{\star}}$ is of the form $B=B_{\Gamma}$, where $\Gamma$ is one of the following even closed walks:

(1) $\Gamma$ is an even cycle of $C^{\star}$,

(2) $\Gamma$ consists of two odd cycles of $C^{\star}$ intersecting in exactly one vertex,

(3) $\Gamma=\left(C,\left\{p_{i}, q_{i}\right\}, C^{\prime}\right)$ where $1 \leq i \leq k$, i.e. it consists of the vertex disjoint odd cycles $C, C^{\prime}$ joined by the edge $\left\{p_{i}, q_{i}\right\}$.

But $C^{\star}$ has no vertex of degree greater than three, so there are no two odd cycles of $C^{\star}$ intersecting in exactly one vertex. Furthermore every primitive binomial of $I_{C^{\star}}$ is also a circuit. Consequently the universal Gröbner basis of $P_{C}$ consists of all binomials of the form $B_{\Gamma}$, where either $\Gamma$ is an even cycle of $C^{\star}$ or $\Gamma=$ $\left(C,\left\{p_{i}, q_{i}\right\}, C^{\prime}\right)$.

Remark 3.15. Let $G$ be a connected non-bipartite graph with exactly one cycle. As Example 3.10 demonstrates, the equality $U\left(P_{G}\right)=\mathcal{C}\left(I_{G^{*}}\right)$ does not hold in general. From Theorem 3.4 in 12 we have that a binomial $B$ belongs to the 
universal Gröbner basis of $P_{G}$ if and only if $B=B_{\Gamma}$, where $\Gamma$ is a mixed even closed walk of $G^{\star}$. For the definition of a mixed walk see [12].

Another interesting case of a bipartite graph is that of a tree $G$. The next theorem provides a graph theoretic characterization of the elements in the universal Gröbner basis of $P_{G}$.

Theorem 3.16. Let $G$ be a tree on the vertex set $\{1, \ldots, n\}$, then the universal Gröbner basis of $P_{G}$ is given by the set

$$
U\left(P_{G}\right)=\left\{B_{\Gamma} \mid \Gamma \text { is an even cycle of } G^{\star}\right\} .
$$

Proof. By the proof of Lemma 3.3 the graph $G^{\star}$ is bipartite and also $P_{G}=I_{G^{\star}}$. So $I_{A_{G}}=I_{G^{*}}$, and therefore we have, from Theorem 2.6. that $U\left(P_{G}\right)=\mathcal{C}\left(I_{G^{*}}\right)$. Using Theorem 3.11 we conclude that the universal Gröbner basis of $P_{G}$ consists of all the binomials $B_{\Gamma}$, where $\Gamma$ is an even cycle of $G^{\star}$.

Remark 3.17. Given a tree $G$, we have that the cardinality of $U\left(P_{G}\right)$ is equal to the number of paths in $G$. Furthermore the maximum degree of a binomial in the universal Gröbner basis of $P_{G}$ equals $l+1$, where $l$ is the length of the longest path of $G$. In particular, for a star graph $G$ on the vertex set $\{1, \ldots, n\}$ with $n \geq 3$, the universal Gröbner basis of $P_{G}$ consists of $\frac{n(n-1)}{2}$ binomials and the maximum degree of a binomial in $U\left(P_{G}\right)$ is equal to three.

Remark 3.18. For a path graph $G$ with $n$ vertices, we have that the maximum degree of a binomial in the universal Gröbner basis of $P_{G}$ equals $n=\left\lfloor\frac{n+(n-1)+1}{2}\right\rfloor$.

\section{REFERENCES}

[1] I. Bermejo, I. Garcia-Marco and E. Reyes, Graphs and complete intersection toric ideals, $J$. Algebra Appl., 14 (2015), no. 9, 1540011, 37 pp.

[2] T. Bogart, R. Hemmecke and S. Petrovic, Universal Gröbner bases of colored partition identities, Exp. Math., 21 (2012), no. 4, 395-401.

[3] J. A. De Loera, Triangulations of polytopes and computational algebra, PhD thesis, Cornell University, 1995.

[4] P. Diaconis, D. Eisenbud and B. Sturmfels, Lattice walks and primary decomposition, in: B. E. Sagan, R. P. Stanley (Eds.), Mathematical Essays in Honor of Gian-Carlo Rota, Birkhäuser, Boston, 1998, pp. 173-193.

[5] V. Ene and A. A. Qureshi, Ideals generated by diagonal 2-minors, Comm. Algebra, 41 (2013), 3058-3066.

[6] J. Morton, Relations among conditional probabilities, J. Symbolic Comput., 50 (2013), 478492.

[7] H. Ohsugi and T. Hibi, Toric ideals generated by quadratic binomials, J. Algebra, 218 (1999), 509-527.

[8] H. Ohsugi and T. Hibi, Toric ideals of finite graphs and adjacent 2-minors, Math. Scand., 114 (2014), no. 2, 185-190.

[9] F. Rapallo, Algebraic Markov bases and MCMC for two-way contingency tables, Scand. J. Statist., 30 (2003), no. 2, 385-397.

[10] A. Schrijver, Theory of Linear and Integer Programming, John Wiley and Sons, New York, 1986.

[11] B. Sturmfels, Gröbner Bases and Convex Polytopes, University Lecture Series, No. 8 American Mathematical Society Providence, R.I. 1995.

[12] C. Tatakis and A. Thoma, On the universal Gröbner bases of toric ideals of graphs, J. Combin. Theory Ser. A, 118 (2011), 1540-1548.

[13] R. H. Villarreal, Monomial Algebras, Marcel Dekker, New York, 2001. 
Department of Mathematics, Mimar Sinan Fine Arts University, Istanbul, 34427, TURKEY

E-mail address: katsabek@aegean.gr 